\title{
Isolated lumbar-4 vertebral cryptococcosis in an immunocompetent patient-A case report and literature review
}

\author{
Tanya Minasian ${ }^{1,2,3,4,5}$, Omid R. Hariri ${ }^{1,2,3,4,5^{*}}$, Casey Corsino ${ }^{2}$, Dan E. Miulli ${ }^{1,2}$, \\ Saman Farr' ${ }^{2}$, Javed Siddiqi $i^{1,2,3,4}$ \\ ${ }^{1}$ Department of Neurosurgery, Arrowhead Regional Medical Center, Colton, USA; *Corresponding Author: ohaririucla@gmail.com \\ ${ }^{2}$ Division of Neurosurgery, Department of Surgery, College of Osteopathic Medicine, Western University of Health Sciences, \\ Pomona, USA \\ ${ }^{3}$ Department of Neurosurgery, Riverside County Regional Medical Center, Moreno Valley, USA \\ ${ }^{4}$ Department of Neurosurgery, Institute of Clinical Orthopaedics \& Neurosciences, Desert Regional Medical Center, Palm Springs, \\ USA \\ ${ }^{5}$ Department of Neurosurgery, Kaiser Permenante Medical Center, Fontana, USA
}

Received 11 July 2013; revised 2 August 2013; accepted 8 August 2013

Copyright (C 2013 Tanya Minasian et al. This is an open access article distributed under the Creative Commons Attribution License, which permits unrestricted use, distribution, and reproduction in any medium, provided the original work is properly cited.

\section{ABSTRACT}

Background: The purpose of this case report is to present an unusual and unique case of vertebral osteomyelitis due to the organism Cryptococcus neoformans, which was found to be isolated to the fourth lumbar vertebra in an immunocompetent patient. Cryptotoccus neoformans is an encapsulated yeast which typically presents in severely immunocompromised patients. Vertebral osteomyelitis is most commonly associated with bacterial infections. Case Description: A 51-year-old male presented with severe pain localized to the lumbar region, and a high grade fever for $\mathbf{1 5}$ days, chills, urinary hesitancy, dysuria, and fatigue. The patient's only past medical history included Type II Diabetes Mellitus. Neoplasms and HIV were ruled out. No source of entry was located upon examination and the lungs were negative for the presence of Cryptococcal pathogen. The CT scan revealed a lytic lesion located in the fourth lumbar vertebral body. A bone biopsy confirmed the presence of Cryptococcus neoformans as the source of infection. A follow up visit was also conducted to examine the patient's status of infection, and for the presence of complications. Conclusion: At this time, it is important to note Cryptococcus neoformans can be isolated to a single vertebral level. This case study is pivotal in demonstrating the importance of the comprehension of rare, and non-traumatic Cryptococcal infections in Central Nervous System, showing also that immunocompetent patients are well at risk for this infectious process.

Keywords: Vertebral Osteomyelitis; Cryptococcus; Immunocompetent; Lytic Lesion; Isolated Infection

\section{INTRODUCTION}

Vertebral osteomyelitis is an infection of the vertebral body, which can progress to abscess formation and spread to adjacent structures hematologically. This can ultimately lead to destruction of intervertebral discs and vertebral bodies, possibly leading to eventual spinal instability, vertebral body collapse, and neural compression. Because the spread of the infection is typically via the blood, multiple loci of infection are common. The most common site of infection is the lumbar spine, with a rate of $45 \%$ - 55\%, followed by thoracic, cervical, and sacral regions [1]. The most common organisms found in vertebral osteomyelitis are Staphylococcus aureus and coagulase negative staphylococci, and occurrences are rarely caused by fungal and parasitic infections [1,2].

Cryptococcus neoformans is a budding yeast surrounded by a polysaccharide capsule that contains antigenic determinants permitting identification. It is distributed worldwide, existing in nature as a soil saprophyte, and is most commonly found in roosting sites of birds, particularly pigeons [3]. Infection results from inhalation of spores, which germinate in pulmonary tissue and may 
disseminate via the bloodstream to the brain, meninges, bone marrow, and skin. Bone involvement, usually osteolytic, is documented in $5 \%-10 \%$ of patients with cryptococcal infection. Differential diagnosis must be determined to rule out neoplastic lesions or osseous tuberculosis [4-6]. Moreover, diabetes mellitus can increase incidence rates of infection with Cryptococcus neoformans. This infection is common in adults with an average age of 60 to 62 years old, affecting males more so than females, with a predominance of 55\% - 75\% [1].

The yeast is known for its opportunistic infection, especially in those who are immunosuppressed and immunocompromised. Infection in the United States prior to 1980 with Cryptococcus neoformans was less than one case per million persons per year. In the 1980's during the AIDS epidemic, the incidence increased to 5\% - 10\% of AIDS patients. With advances in antiretroviral therapy, the annual incidence rate has decreased to seven cases per 1000 people in the year 2000 [7]. It is very rare for immunocompetent patients to be afflicted with this disease, with amounts estimated to be 0.2 per million per year [2]. There have been few documented cases reporting thoracic vertebral cryptococcus with cord compression [8]. However, to our knowledge and extensive research, there have been no previously documented presentations of an isolated lumbar vertebral cryptococcosis [9].

We are reporting a case of an unusual presentation of a pathologically proven case of isolated cryptococcosis infection to a single level lumbar vertebral body in an immunocompetent patient.

\section{CASE REPORT}

A 51-year-old Pakistani male presented with low back pain and fever for 15 days associated with chills and night sweats. Other symptoms reported include urinary hesitancy, dysuria, and fatigue. He described the severity of his pain as 10/10, sharp, increasing with movement, and located in the mid-lumbar region. However, he denied any weight loss, headaches, dizziness, blurred vision, cough, or nasal discharge. He also denied sore throat, neck stiffness, chest pain, hematuria, or loss of balance. The patient denied pain, paresthesias, or weakness radiating into the bilateral lower extremities, saddle anesthesia, or bladder or bowel incontinence.

The patient's past medical history was significant for Type II Diabetes Mellitus for ten years and he had been on Metformin. The patient reported chewing tobacco for 16 years with no use in the previous 10 years. The patient lived in Virginia and was employed as a truck driver. Patient denied illicit drug use.

Upon physical examination, the patient was awake, alert, and oriented to person, place, time, and event. Vital signs were stable except sinus tachycardia. No lympha- denopathy was noted. His exam was significant for tenderness to palpation along Lumbar 2, 3, 4 spinous processes with no tenderness to percussion. Normal muscle tone was present, and no atrophy noted. No evidence of trauma to the lumbar region. He had 5/5 muscle strength bilaterally in his upper and lower extremities. Normal deep tendon reflexes were noted, without any long tract signs. Rectal exam was intact.

Differential diagnosis for a 51-year-old immunocompetent male presenting with an isolated lumbar vertebral body lytic lesion must include an infectious etiology, but given the location of the lesion in addition to the patient's complaint of urinary hesitancy/urgency, a metastatic lesion from the prostate to the vertebral body must be excluded.

Laboratory workup revealed a WBC count of 8.2, HgB of 14.1, Hct of 42.1, and Platelet count of 240. ESR and CRP values were elevated, 77 and 4.26, respectively. His tumor markers (AFP, CEA, CA 19-9, CA 125, B-HCG, and PSA) were all negative. His HIV and Hepatitis panel were also negative. Blood cultures were negative on three separate occasions. His urinalysis was positive for $2+$ glucose but urine culture was negative.

CSF was clear with WBC 61, RBC 3, Lymphocytes 100, Glucose 92 and Protein 81. His Cryptococcus antigen, Histoplasmosis, and India Ink all came back negative. Microbiology culture was positive for Cryptococcus neoformans. The patient was found to have a negative protein electrophoresis for multiple myeloma.

A CT chest/abdomen/pelvis was performed and no masses or other sources of primary neoplasm were identified. Other studies including dedicated spine CT were performed and a lytic lesion was identified in the L4 vertebral body. There was no evidence of canal compromise on MR imaging. An Interventional Radiology CT guided L4 vertebral lytic lesion biopsy was performed. Microsections demonstrated diffuse acute and chronic inflammatory exudate with non-caseating granulomas. No normal hematopoiesis tissue was present. There were numerous small to intermediate size encapsulated yeast forms occurring singly and occasionally as narrow-based budding yeasts. The microorganisms were morphologically suggestive of Cryptococcus by fungal stain (GMS with adequate controls) (Figure 1).

\section{DISCUSSION}

Certain predisposing factors make the vertebral column a suitable site for infection, including the lack of epiphyseal growth, voluminous, yet slow blood supply, and the presence of bone marrow within the vertebral body. Approximately $40 \%$ of the cases of vertebral osteomyelitis lack an obvious port of entry [1].

Cryptococcus can infect any organ in the body, but has a predilection for the lung and central nervous system 


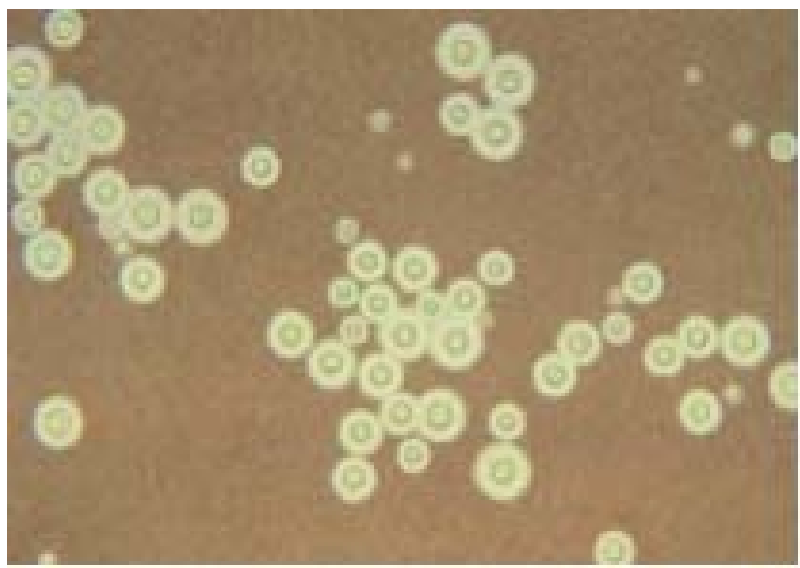

Figure 1. The above photograph is the result of the patient's biopsy from the IR guided L4 vertebral body lytic lesion. Microsections demonstrate diffuse acute and chronic inflammatory exudate with non-caseating granulomas. No normal hematopoiesis tissue is present. There are numerous small to intermediate size encapsulated yeast forms occurring singly and occasionally as narrow-based budding yeasts. The microorganisms are morphologically suggestive of cryptococcus by fungal stain (GMS with adequate controls).

and travels via the bloodstream [7]. Symptoms can range from asymptomatic colonization to severe pneumonia.

Cryptococcal vertebral infection can be treated medically with anti-fungal agents including a six week course of amphotericin B, fluconazole, and flucytosine [10]. Biopsy with demonstration of the infectious organism is the gold standard for diagnosis. Approximately only $30.4 \%$ of patients who have radiographic evidence of infection will have positive blood cultures [11]. Surgical intervention is only warranted if there are neurological symptoms indicating cord compression [1]. In immunocompetent patients, relapse of infection has not been documented to our knowledge. However, in immunocompromised and immunosuppressed persons, relapse is common. Long-term maintenance treatment with fluconazole has prevented relapse in patients with AIDS [8].

\section{CONCLUSION}

The patient presented in this case study returned for a follow up visit post treatment of a six week course of intravenous amphotericin B, fluconazole, and flucytosine and was found to have no signs of recurrent infection or complications. Upright X-rays were not suggestive of any vertebral column instability. The pain had improved and the patient was doing well. There have been no other documented cases of isolated cryptococcal infection involving only a single vertebral body in the lumbar vertebral region.

\section{REFERENCES}

[1] Jaramillo-de la Torre, J.J., et al. (2006) Vertebral osteomyelitis. Neurosurgery Clinics of North America, 17, 339-351. doi:10.1016/j.nec.2006.05.003

[2] Houda, B., et al. (2011) Vertebral cryptococcosis in an immunocompetent patient-A case report. Pan African Medical Journal, 8, 42.

[3] Chae, H.S., et al. (2012) Rapid direct identification of Cryptococcus neoformans from pigeon droppings by nested PCR using CNLAC1 Gene. Poultry Science, 91, 1983-1989. doi:10.3382/ps.2012-02307

[4] Bubb, H. (1955) Cryptococcus neoformans infection in bone. South African Medical Journal, 29, 1259-1261.

[5] Jesse, C.H. (1947) Cryptococcus neoformans infection (torulosis) of bone; report of a case. Journal of Bone \& Joint Surgery, 29, 810.

[6] Schiappa, D., et al. (2002) An auxotrophic pigmented Cryptococcus neoformans strain causing infection of the bone marrow. Medical Mycology, 40, 1-5.

[7] Bicanic, T. and Harrison, T. (2004) Cryptococcal meningitis. British Medical Bulletin, 72, 99-118. doi:10.1093/bmb/ldh043

[8] Mitchell, T.G. and Perfect, J.R. (1995) Cryptococcosis in the era of AIDS-100 years after the discovery of Cryptococcus neoformans. Clinical Microbiology Reviews, 8, 515-548.

[9] Buchanan, K. and Murphy, J. (1998) What makes Cryptococcus neoformans a pathogen. Emerging Infectious Diseases, 4, 71. doi:10.3201/eid0401.980109

[10] Matsushita, T. and Suzuki, K. (1985) Spastic paraparesis due to cryptococcal osteomyelitis. A case report. Clinical Orthopaedics and Related Research, 196, 279-284.

[11] Sehn, J. and Gilula, L. (2012) Percutaneous needle biopsy in diagnosis and identification of causative organisms in cases of suspected vertebral osteomyelitis. European Journal of Radiology, 81, 940-946. doi:10.1016/j.ejrad.2011.01.125 